\title{
Evolution of target condition in reactive HiPIMS as a function of duty cycle: An opportunity for refractive index grading
}

\author{
Rajesh Ganesan, ${ }^{1,2}$ Behnam Akhavan, ${ }^{1}$ James G. Partridge, ${ }^{3}$ Dougal G. McCulloch, ${ }^{3}$ \\ David R. McKenzie, ${ }^{1, a)}$ and Marcela M. M. Bilek ${ }^{1, b)}$ \\ ${ }^{1}$ School of Physics, University of Sydney, Sydney, New South Wales 2006, Australia \\ ${ }^{2}$ Nanostructured Thin Films and Coatings, Swiss Federal Laboratories for Materials Science and Technology, \\ Duebendorf 8600, Switzerland \\ ${ }^{3}$ School of Applied Science, RMIT University, GPO Box 2476 V, Melbourne, Victoria 3001, Australia
}

(Received 4 November 2016; accepted 17 January 2017; published online 7 March 2017)

\begin{abstract}
Competition between target erosion and compound layer formation during pulse cycles in reactive HiPIMS opens up the possibility of tuning discharge conditions and the properties of deposited films by varying the duty cycle in situ without altering the reactive gas mixture. Three different reactive systems, hafnium in oxygen, tungsten in oxygen, and tungsten in oxygen/nitrogen, are studied in which amorphous films of hafnium oxide $\left(\mathrm{HfO}_{2}\right)$, tungsten oxide $\left(\mathrm{WO}_{3}\right)$, and tungsten oxynitride $\left(\mathrm{WO}_{\mathrm{x}} \mathrm{N}_{\mathrm{y}}\right)$ are deposited. We show that the cyclic evolution of the target surface composition depends on the properties of the target including its affinity for the reactive gas mix and the compound layer melting point and volatility. We find that pulse length variations modulate the target compound layer and hence the discharge chemistry and properties of the films deposited. The refractive indices of $\mathrm{HfO}_{2}$ and $\mathrm{WO}_{3}$ were progressively reduced with the duty cycle, whereas that of $\mathrm{WO}_{\mathrm{x}} \mathrm{N}_{\mathrm{y}}$ increased. These variations were found to be due to changes in the chemical composition and/or densification. We present and validate a phenomenological model that explains these findings in terms of a compound layer on the target surface that undergoes evolution during each pulse resulting in a cyclic equilibrium. The end points of the composition of the target surface depend on the duty cycle. Tuning the pulse characteristics holds great promise for the fabrication of multilayer films with through thickness graded properties. Published by AIP Publishing.

[http://dx.doi.org/10.1063/1.4977824]
\end{abstract}

\section{INTRODUCTION}

High Power Impulse Magnetron Sputtering (HiPIMS) is a pulsed DC sputtering technique, in which high power is applied to the magnetron target in unipolar pulses at a low duty cycle and a low repetition rate to maintain a manageable average output power. ${ }^{1-5}$ The high power pulses generate a high-density plasma in which $40 \%-80 \%$ of the sputtered target material is ionized. ${ }^{6-10}$ HiPIMS has been a topic of interest over the past decade, as it combines the advantages of magnetron sputtering with those of an ionized depositing flux..$^{2,11}$ The presence of ions is favorable as substrate bias can be used to control the kinetic energy of the depositing flux, allowing control of the microstructure of the films and improving adhesion to the substrate. ${ }^{12,13}$

In reactive HiPIMS, metal targets are sputtered by argon mixed with reactive gases $\mathrm{O}_{2} / \mathrm{N}_{2}$ to deposit metal oxides/ nitrides with functional properties for optical and electronic applications. ${ }^{14-18}$ Films grown by reactive HiPIMS require a lower deposition temperature and exhibit improved functional properties such as higher refractive index and lower surface roughness when compared to those deposited by standard direct current magnetron sputtering (dcMS). ${ }^{19}$ The reactive gases that exist generally in molecular states undergo enhanced dissociation due to the high electron densities in

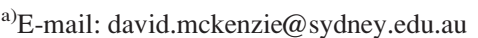

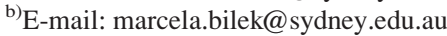

HiPIMS, which is beneficial for the deposition of high quality oxides and nitrides with enhanced functionalities. ${ }^{20-25}$

Oxides of hafnium are widely used in the electronics industry. ${ }^{15,16,26}$ Hafnium oxide is a superior material for high- $\kappa$ dielectrics due to its attractive properties. These properties include a large bandgap $(>5.6 \mathrm{eV})$, high dielectric permittivity $(\kappa>16),{ }^{27}$ high thermal stability in contact with silicon, and high energy barriers with respect to silicon that restrict the movement of free charges. ${ }^{28}$ Tungsten oxide and tungsten oxynitride have attractive applications in sensing, ${ }^{29}$ electronic, ${ }^{30}$ electrochromic, ${ }^{31}$ and electrochemical devices. ${ }^{32}$

In magnetron sputtering, a compound layer forms on the target, a phenomenon known as "target poisoning,"33-36 which often results in a significantly reduced sputter yield and, thereby, a reduced deposition rate. Hysteresis occurs when a single gas flow value does not give a stable sputtering outcome, and as a result, the gas flow or electrical power input to the discharge must be continually adjusted to maintain the desired deposition conditions. Hysteresis in magnetron sputtering of tungsten oxide leads to substoichiometric regions in the deposited films. ${ }^{37}$ In a pulsed sputtering process, such as HiPIMS, a compound layer forms on the target during the pulse off time, and the compound layer is eroded during the high voltage pulse. Hysteresis-free operation is therefore possible by selection of an optimal duty cycle, ${ }^{19,38,39}$ and the influence of pulse characteristics on the properties of deposited films has also been demonstrated. Despite its proven 
success in depositing compound thin films, the mechanism of the target-plasma interaction in reactive-HiPIMS and its correlation to the film properties are still not understood. In this work, we gain understanding by varying the duty cycle applied in reactive $\mathrm{Ar} / \mathrm{O}_{2}$ HiPIMS discharges of hafnium and tungsten. The variation in discharge characteristics and optical/electronic film properties as a function of duty cycle reveals the important role of the evolution of target surface chemistry. We develop a phenomenological model describing the HiPIMS deposition of hafnium oxide, tungsten oxide, and tungsten oxynitride that builds on a previous model. ${ }^{38-40}$ Our new model explains the role of the compound layer on the target surface and the dependence of its behavior on the widely differing melting points and volatilities of these compounds.
This work reveals the potential to use duty cycle control for the deposition of multilayer films with graded refractive indices. Duty cycle control would provide a simple and cost effective refractive index modulation technique for industrial fabrication of graded optical coatings.

\section{EXPERIMENTAL METHODS}

All coatings were deposited using a vacuum deposition system (AJA International $1800 \mathrm{~F}$ ) schematically illustrated in Figure 1(a). The depositions were carried out in a reactive atmosphere of various mixtures of ultra-high purity (99.9995\%) $\mathrm{O}_{2}, \mathrm{~N}_{2}$, and Ar (BOC Australia) gases. Hafnium and tungsten sputtering targets (purity $=99.995 \%$, American

(a)

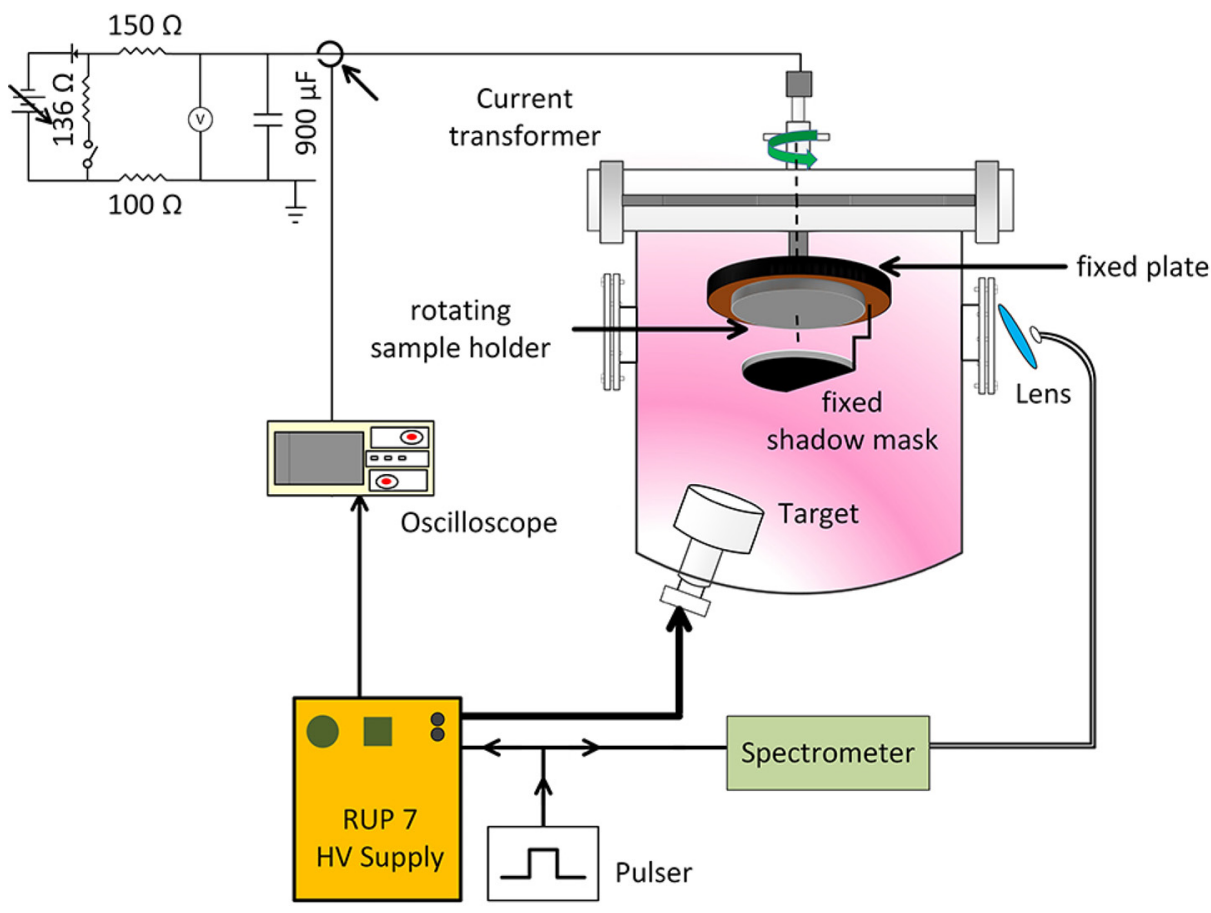

FIG. 1. (a) Top panel shows a schematic diagram of the experimental setup. (b) The calculated refractive index for three different estimations of thickness for the hafnium oxide film, for which the actual thickness is $13.9 \mathrm{~nm}$ (deposited for $10 \mathrm{~min}$ with the $100 \mu$ s long pulses). Substrate interference features are evident for the incorrect thicknesses.

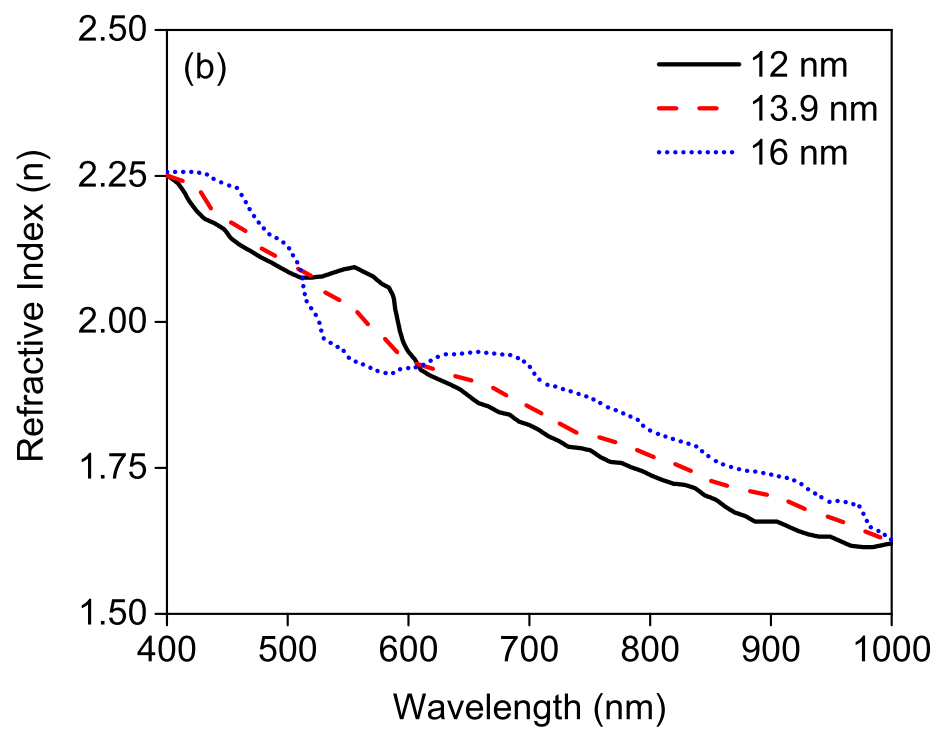


Elements) of $75.6 \mathrm{~mm}$ diameter and $6.35 \mathrm{~mm}$ thickness were utilized in an unbalanced magnetron gun in which the magnetic field strength of the central magnet was $10 \%$ weaker than that of the outer magnets. The magnetron was energized by a RUP7 pulsed power supply (GBS Electronik GmbH) delivering the voltage pulses of 450 and $825 \mathrm{~V}$ for the reactive HiPIMS of hafnium and tungsten, respectively. The ntype $\mathrm{Si}\left(\begin{array}{lll}1 & 0 & 0\end{array}\right)$ substrate with a $\mathrm{SiO}_{2}$ layer was placed $19 \mathrm{~cm}$ away from the target, inclined at $33^{\circ}$ to the normal. The substrate holder was coupled to a $900 \mu \mathrm{F}$ capacitor, allowing a self-bias to form. Pulse lengths were varied from 80 to $200 \mu \mathrm{s}$ in increments of $20 \mu \mathrm{s}$. The pulse frequency was kept constant at $150 \mathrm{~Hz}$. Each deposition was carried out for $10 \mathrm{~min}$ for each pulse length condition at an unchanged total gas pressure of 2.8 mTorr. Equal flow rates of argon and oxygen (10 sccm each) were supplied for the reactive HiPIMS of tungsten and hafnium. Deposition of tungsten oxynitride films was carried out with the gases $\mathrm{Ar} / \mathrm{O}_{2} / \mathrm{N}_{2}$ supplied with the flow rates of 10:5:5 sccm, respectively. Seven samples were mounted along a curve on the substrate holder that could precisely rotate through a mechanical feedthrough. A shadow mask, fixed between the target and the substrate, was used to reveal the samples for each deposition. The distance between the planes of the shadow mask and the substrate was adjusted to $0.75 \mathrm{~mm}$. After each deposition condition, the samples were shaded one after another using the shadow mask to prevent them from collecting the deposits arriving from the subsequent deposition condition. No arcing was observed during the deposition. The current and voltage waveforms were recorded by a digital oscilloscope (Agilent model: DSOX-2014 A). The current-voltage waveforms for reactive HiPIMS of tungsten was recorded in average mode, in order to show the dependent subtle differences in the waveforms by reducing noise and increasing vertical resolution in the recording signal.

Time-resolved optical emission spectroscopy (OES) was used to monitor the plasma species with a lens focused on the target surface using an Acton Research Corporation SpectroPro 2750 spectrometer. Outside a viewport on the chamber, a lens with a focal length of $50 \mathrm{~mm}$ was used to image a section of the racetrack region of the target surface onto the entrance of a fused-silica optical fiber bundle with a diameter of $1 \mathrm{~mm}$. The other end of the fiber bundle directed the light along the length of a $50 \mu \mathrm{m}$ entrance slit attached to a spectrograph. The grating used had 1200 lines $\mathrm{mm}^{-1}$, resulting in a nominal resolution of $0.0140 \mathrm{~nm}$ at $300 \mathrm{~nm}$ and $0.0120 \mathrm{~nm}$ at $750 \mathrm{~nm}$. The timeresolved spectra were acquired for an acquisition time of $250 \mathrm{~ns}$, with frame intervals of $5 \mu \mathrm{s}$. At each time point, the emission intensity was averaged for 150 pulses.

$\mathrm{X}$-ray photoelectron spectroscopy (XPS) to determine film compositions was performed using a Thermo Scientific $\mathrm{K}-\mathrm{Alpha}$ spectrometer with an $\mathrm{Al} \mathrm{K} \alpha$ source $(1486.7 \mathrm{eV})$. The nitrogen content in the tungsten oxynitride films was determined by XPS after using xenon to sputter away the surface layer. To verify that preferential sputtering did not affect the depth profiles, two different energies (100 and $200 \mathrm{eV})$ were used for five time points $(20,40,60$, and $100 \mathrm{~s})$ at different sites on the film surface. Consistency of the measured composition indicated that the profiles were not affected by preferential sputtering.
XPS depth profiling measurements of the target surfaces were performed using a Quantum 2000 Scanning ESCA microscope. The surface was bombarded with $2.5 \mathrm{keV} \mathrm{Ar}^{+}$ ions, and the argon ion beam was focused over an area of $2 \times 2 \mathrm{~mm}$ around the point of measurement. The $\mathrm{C}-\mathrm{C}$ peak at $284.8 \mathrm{eV}$ was used for calibration of the spectra.

The thickness and optical properties of the films were determined using a spectroscopic ellipsometer in the wavelength range of 192.5-1000 nm (2000D, JA Woollam Co.) by modelling the data obtained from ellipsometry using the method described by Arwin and Aspnes. ${ }^{41-43}$ This method requires the presence of strong interference features for accurate determination of the optical properties. Therefore, a silicon dioxide $\left(\mathrm{SiO}_{2}\right)$ layer with a thickness of $500 \mathrm{~nm}$ was deposited on the silicon wafers using reactive HiPIMS of Si. The $\mathrm{SiO}_{2}$-coated silicon wafers were used as substrates throughout this study. The optical constants for the deposited layer were calculated for several estimates for a film thickness. The correct thickness can then be determined as the one for which the plot of optical constants as a function of photon energy does not show evidence of the interference feature from the substrate $(13.9 \mathrm{~nm}$ in Figure 1(b)).

The optical band gap $\left(E_{g}\right)$ of amorphous hafnium oxide films, assumed to be direct, was determined by extrapolating the straight line obtained from the deflection points of the curves $(\alpha \mathrm{h} \gamma)^{2}$ plotted against the photon energy (h $\left.\nu\right)$ using the Tauc relation. ${ }^{44}$ The absorption coefficient $(\alpha)$ was calculated from the extinction coefficient $(k)$ according to the relation $\alpha=\frac{4 \pi k}{\lambda}$.

The radius of the substrate curvature was measured by using an optical profilometer (WYKO NT1100) on both sides of the sample (mirror polished and unpolished) before and after film deposition. The stress was then determined from the measured curvature using Stoney's equation especially derived $^{45}$ for single crystalline Si (100) wafers as given below

$$
\sigma_{f}=\frac{t_{s}}{6 \cdot R \cdot t_{f} \cdot\left(S_{11}^{S i}+S_{12}^{S i}\right)}
$$

in which, $t_{f}$ is the thickness of the film, $t_{s}$ is the thickness of the substrate, and $R$ is the radius of curvature of the substrate after deposition of the film. The terms $S_{11}^{S i}$ and $S_{12}^{S i}$ are elements of the compliance tensor of silicon, and the biaxial modulus $M_{001}^{S i}=1.803 \times 10^{11} \mathrm{~Pa}$ was obtained from the factor $\frac{1}{\left(S_{11}^{S i}+S_{12}^{S i}\right)}$.

For electrical characterization, samples of uniform thickness $(75 \mathrm{~nm})$ were made for each of the deposition conditions. The $0.35 \mathrm{~mm}$ diameter silver electrical contacts for metal-insulator-semiconductor (MIS) capacitor structures were formed on the deposited samples using DCMS to a thickness of $40 \mathrm{~nm}$. The through film leakage current was measured using an HP4155A parameter analyzer.

\section{RESULTS}

\section{A. Characterization of the reactive sputtering process}

Figure 2 shows characteristic profiles of target current during the HiPIMS voltage pulse measured at the power 

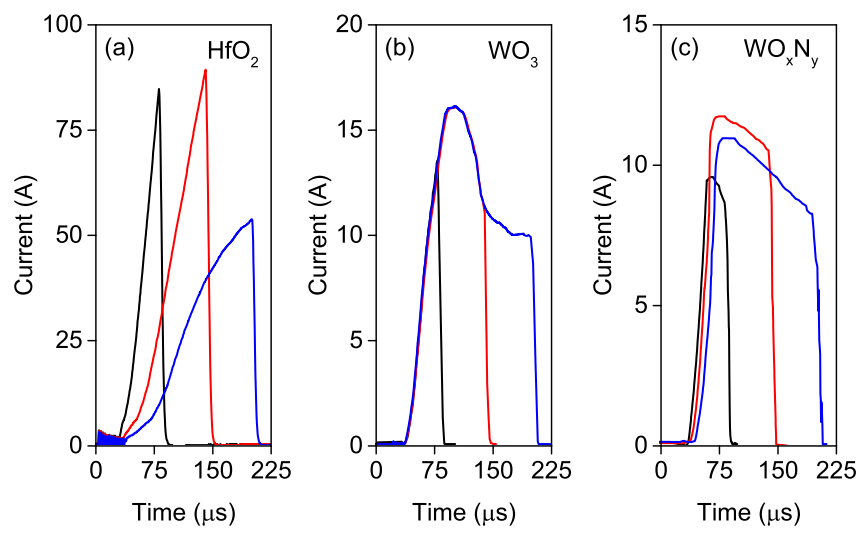

FIG. 2. The target current as a function of time for reactive HiPIMS of (a) hafnium sputtered in oxygen, (b) tungsten sputtered in oxygen, and (c) tungsten sputtered in an oxygen-nitrogen reactive gas mixture.

supply output as a function of time for the discharge of hafnium and tungsten targets. Due to the pulsed nature of the discharge, the target surface is alternately eroded by bombarding ions (during the HiPIMS pulse) and subject to reaction with the sputtering atmosphere and afterglow plasma (between HiPIMS pulses). Hence, the target is less oxidized at the end of a pulse compared to at the pulse onset. The dependence of the onset delay on the pulse length of the discharge on the hafnium target (Figure 2(a)) is attributed to the difference in the secondary electron emission coefficient between the oxidized (poisoned) and metallic target surfaces. ${ }^{38,40}$ HiPIMS using a tungsten target in a reactive $\mathrm{O}_{2}$ atmosphere (Figure 2(b)) does not show any dependence of the onset of discharge current on the pulse length. The secondary electron emission coefficient of an oxidized tungsten surface is higher than the clean metal surface, ${ }^{46}$ so the fact that there is no difference in the onset delay time or rate of increase in the target current after onset indicates that the condition of the target surface is the same regardless of which pulse length is used. For pulse lengths of over $100 \mu \mathrm{s}$, the current reaches a peak value and begins to fall due to the composition change in the plasma to include more sputtered atoms and the expulsion of gas by momentum transfer, gas heating, and expansion. Such a gas rarefaction process is also exhibited for the reactive HiPIMS of tungsten, where nitrogen is included in a reactive gas mixture (Figure 2(c)) as indicated by the current peak and subsequent reduction. In this case, the current onset delay depends on the pulse length, as for the hafnium target, suggesting that the target surface condition also varies with the pulse length when nitrogen is included and that the secondary electron emission coefficient for the oxynitride surface is higher than that for the clean metal surface.

The different behavior observed at the onset of the pulses shows that it is the target condition rather than the gas depletion that determines the initial shape of the IV characteristic. This follows because although the reactive gas may be depleted later in the pulse, the off-time is sufficient to allow the gas to be fully replenished by the beginning of the subsequent pulse. Changes in the background gas affect the evolution of the discharge only at later times in the pulse.

Figures 3(a) and 3(b) show the time-resolved ratio optical emission intensity from neutral oxygen to that from hafnium (a) and tungsten (b) based on measurements of three emission lines: neutral oxygen (O I) at $777.19 \mathrm{~nm}$ (upper energy level at $10.74 \mathrm{eV}$ ), neutral hafnium (Hf I) at $417.43 \mathrm{~nm}$ (upper energy level at $3.26 \mathrm{eV}$ ), and neutral
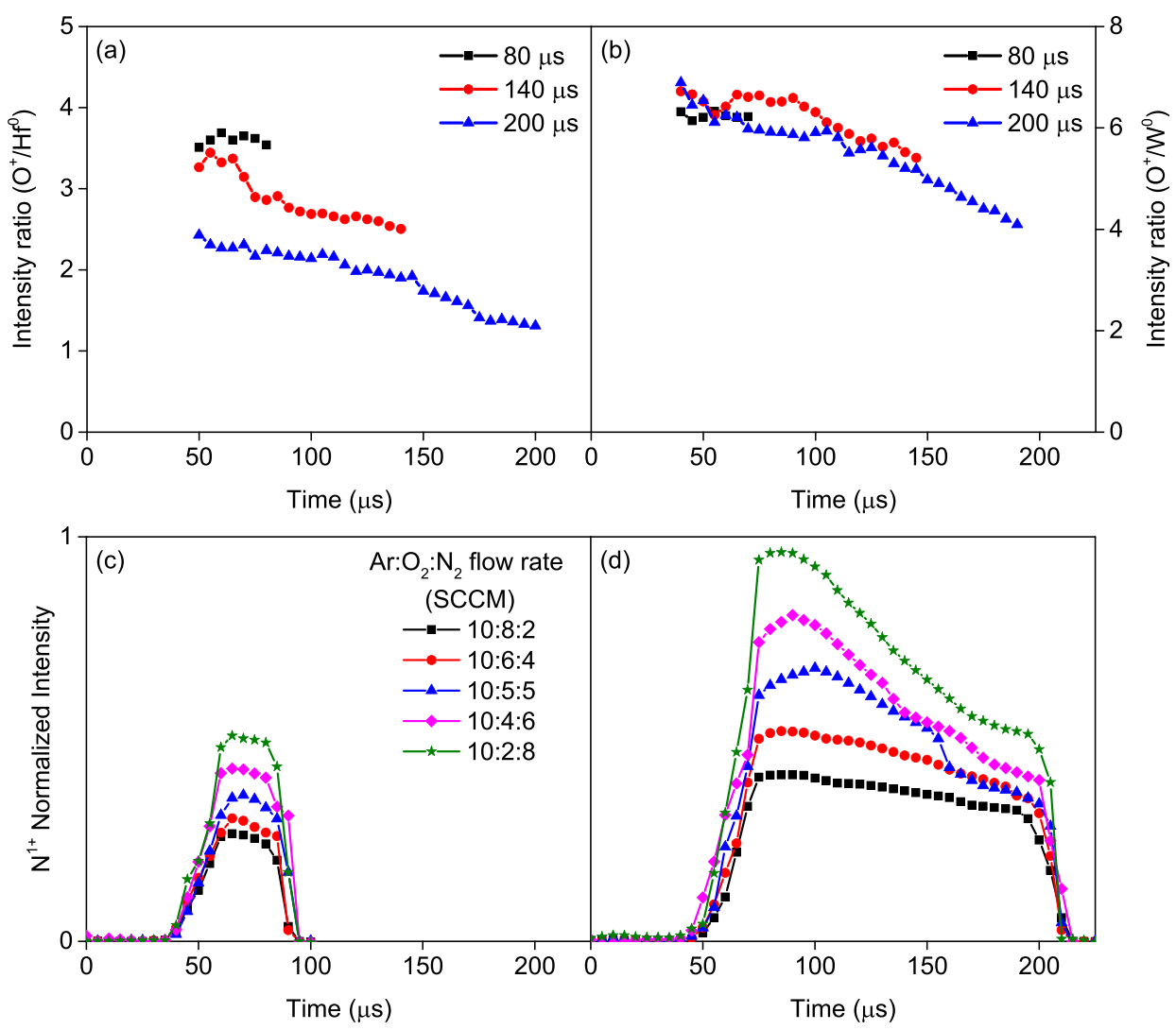

FIG. 3. Top panels show the oxygen ion to metal neutral intensity ratios determined by the optical emission spectroscopy of reactive HiPIMS of hafnium (a) and tungsten (b) in oxygen reactive gas. The oxygen to metal ratio was always higher for tungsten and pulse length dependent for Hf compared to $\mathrm{W}$. The bottom panels show the time evolution of the nitrogen ion emission line at $744 \mathrm{~nm}$ recorded for pulse lengths of $80 \mu \mathrm{s}$ (c) and $200 \mu \mathrm{s}$ (d) for various nitrogen partial pressures as indicated. The flow rates of gases given in the ratios are in units of standard cubic centimeters per minute (sccm). Error bars determined by the statistical distribution of the measurements are contained within the data points. 

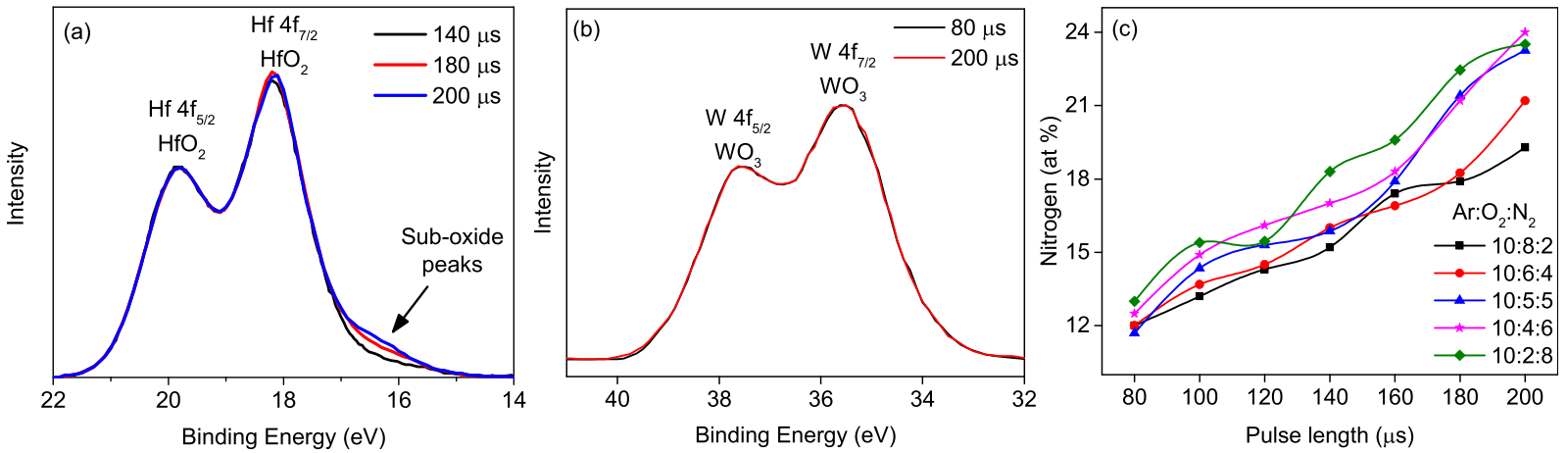

FIG. 4. High resolution X ray photoelectron spectra of (a) the Hf $4 \mathrm{f}$ peak from hafnium oxide films and (b) the W 4f peaks from tungsten oxide films for various pulse lengths as indicated. (c) The nitrogen concentration in atomic \% as a function of pulse length obtained from XPS survey scans for films deposited at the reactive $\mathrm{O}_{2} / \mathrm{N}_{2}$ HiPIMS of tungsten with a range of gas mixture partial pressures. Argon to oxygen to nitrogen flow rate ratios are indicated.

tungsten (W I) at $429.4 \mathrm{~nm}$ (upper energy level at $3.25 \mathrm{eV}$ ). The ratios of line intensities enable comparison of changes in the relative populations when emission lines with comparable upper level energies are used. The overall intensity of emission from all species varies according to the shape of the current pulse. In the case of the Hf discharge, there is a strong dependence of this ratio on the pulse length, indicating that the proportion of oxygen and hafnium species in the discharge is strongly affected by the duty cycle. The relative concentration of oxygen species decreases with increasing pulse length. In contrast, the relative level of oxygen in the reactive discharge with tungsten does not depend on the pulse length, consistent with an unchanging target surface condition as suggested by the target current traces in Figure 2(b). The time resolved studies of the emission line intensities from nitrogen ions (Figures 3(c) and 3(d)) show that the concentration of nitrogen ions is higher for the longer pulses (left panel-200 $\mu \mathrm{s}$ ) than for the shorter ones (right panel$80 \mu \mathrm{s})$. For the gas flow conditions with the highest nitrogen partial pressure (Ar-10: $\mathrm{O}_{2}-2: \mathrm{N}_{2}-8 \mathrm{SCCM}$ ), the intensity of emission from nitrogen ions for the pulse length of $200 \mu$ s is about 2-fold higher than that obtained for the pulse length of $80 \mu \mathrm{s}$. This suggests an increased concentration of nitrogen in the compound layer of the tungsten target with increasing pulse length consistent with a changing target surface composition. We propose that the inclusion of nitrogen has reduced the rate of formation of a compound layer on the target as detailed in the phenomenological model presented in the Discussion section.

\section{B. Film deposition under pulse length control}

Figures 4(a) and 4(b) show high resolution scans of Hf $4 \mathrm{f}$ and $\mathrm{W} 4 \mathrm{f}$ peaks for the films deposited for various pulse lengths. In the case of hafnium oxide films, pulse lengths greater than $160 \mu$ s show the appearance of sub-oxide peaks, clearly visible at the shoulders of the major $\mathrm{Hf}_{4} \mathrm{f}_{7 / 2}$ peak, as indicated by the arrow. This sub-stoichiometry is also evident from the increased intensity of the $4 \mathrm{f}_{7 / 2}$ peak with respect to the $4 f_{5 / 2}$ peak. In contrast, the tungsten oxide films (Figure 4(b)) did not exhibit any sub-stoichiometry. Substoichiometry is consistent with the OES results and indicative of a changing target surface condition in the case of $\mathrm{Hf}$ and a constant target surface condition in the case of $\mathrm{W}$.
Figure 4(c) shows the nitrogen atomic concentration as a function of pulse length for a range of gas mixtures as indicated. Although the nitrogen concentration increases as expected with nitrogen partial pressure in the gas mix, it increases even more strongly with pulse length. This behavior also correlates well with the OES data and suggests that nitrogen is progressively enriched in the target surface layer as the pulse length increases. The atomic composition of films made with equal oxygen and nitrogen flow rates shown in Table I shows that the increase in nitrogen is accompanied by a loss of oxygen, whereas the tungsten content remains approximately constant.

XRD measurements show that both $\mathrm{HfO}_{2}$ and $\mathrm{WO}_{3}$ films are amorphous (Fig. 5). This may be due to the high crystallization temperatures, $535^{\circ} \mathrm{C}$ (Ref. 47) and $>300{ }^{\circ} \mathrm{C},{ }^{48}$ respectively. The $\mathrm{WO}_{3} \mathrm{~N}_{3-\mathrm{x}}$ films are also amorphous. This may be related to the observation that no crystalline phase appears in WNx films with a low nitrogen content. ${ }^{49}$

Figure 6 shows the deposition rates and stress measured from deposited films of hafnium oxide, tungsten oxide, and tungsten oxynitride as a function of pulse length. The deposition rate increases with pulse length in all cases, and the stress is compressive. For the oxides, the stress reaches a maximum and then declines with pulse length. In the case of $\mathrm{Hf}$, the increase in the slope of the deposition rate occurs as the films become sub-stoichiometric, consistent with the increased metallic nature of the target surface. ${ }^{38,40}$ The stress maximum occurs at pulse lengths where the films start to become sub-stoichiometric, consistent with stress relief

TABLE I. Chemical compositions (given in atomic \%) of the tungsten oxynitride films prepared at different HiPIMS pulse lengths using a tungsten target in a gas mixture of oxygen and nitrogen with equal flow rates $\left(\mathrm{Ar}: \mathrm{O}_{2}: \mathrm{N}_{2}=10: 5: 5 \mathrm{sccm}\right)$.

\begin{tabular}{lccc} 
Pulse length $(\mu$ s). & Tungsten (at. \%). & Oxygen (at. \%). & Nitrogen (at. \%). \\
\hline 80 & 27.74 & 60.56 & 11.70 \\
100 & 28.22 & 57.43 & 14.35 \\
120 & 28.30 & 56.40 & 15.30 \\
140 & 28.29 & 55.84 & 15.87 \\
160 & 29.46 & 51.71 & 18.84 \\
180 & 30.52 & 46.65 & 22.83 \\
200 & 29.82 & 46.61 & 23.57 \\
\hline \hline
\end{tabular}




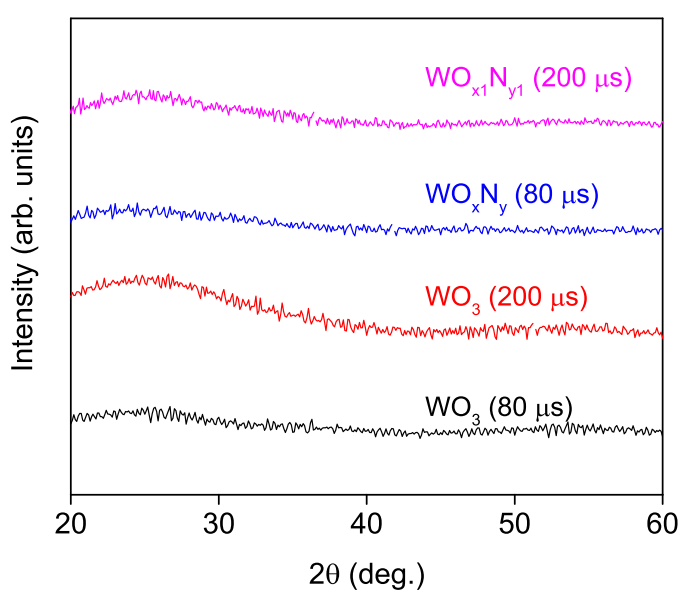

FIG. 5. The XRD patterns of tungsten oxide films and tungsten oxynitride films. All the films deposited over the range of pulse lengths from 80 to $200 \mu$ s are amorphous.

provided by the plastic flow resulting from an increased metallic fraction of lower yield strength. In the case of $\mathrm{WO}_{3}$, the rate of increase in the deposition rate decreases at long pulse lengths when the contribution of deposition occurring under gas rarified conditions becomes significant. Stress

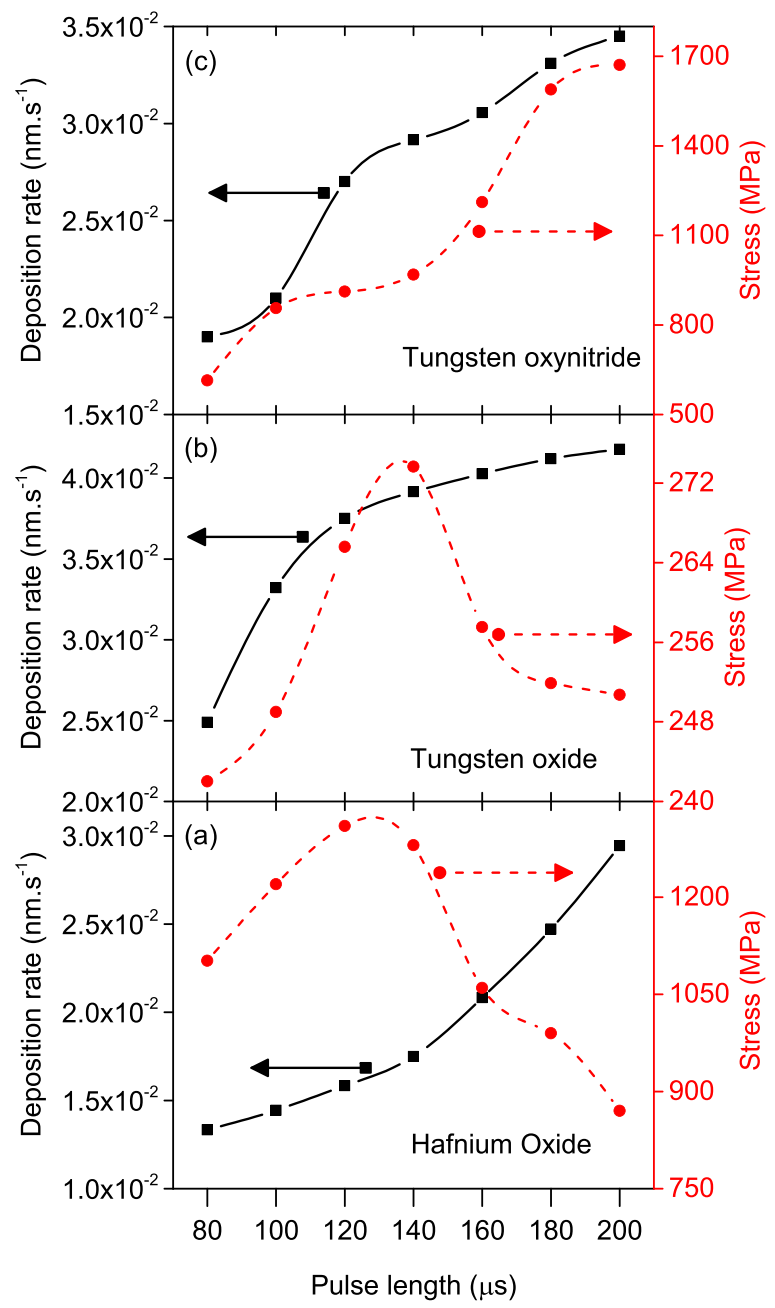

FIG. 6. The deposition rate and the stress measured for (a) hafnium oxide, (b) tungsten oxide, and (c) tungsten oxynitride films as a function of pulse length. increases initially and then decreases when gas rarified conditions dominate. This may be due to stress relief caused by increased energy associated with ions in the reduced background gas pressure resulting from gas rarefaction. The increased energy arises from reduced gas scattering and may cause stress relief. Ion induced stress relaxation is known to be higher for higher energy ions. ${ }^{50}$

In addition, the flux and energy of ions may increase as a result of negative oxygen ion formation. ${ }^{51,52}$ For tungsten oxynitride, the deposition rate increases smoothly with pulse length as would be expected as a result of increasing duty cycle alone. Stresses of tungsten oxynitride films are also compressive and show a progressive increase with pulse length. We attribute this to the increasing nitrogen content of the films, which causes an increase in the toughness and elastic modulus of the film, enabling it to sustain higher levels of compressive stress. For example, the indentation hardness of tungsten nitride is approximately $32 \mathrm{GPa},{ }^{53,54}$ while the indentation hardness of tungsten oxide is $5-7 \mathrm{GPa}^{55,56}$

Both the refractive index and the optical bandgap decrease for the hafnium oxide for pulse lengths longer than $160 \mu \mathrm{s}$, which is attributed to the increasingly sub-stoichiometric composition of the films. ${ }^{38,40}$ For tungsten oxide, although the refractive index decreases, no variation in the optical bandgap was observed. There is no change in the extinction coefficient of the tungsten oxide films, consistent with the stable stoichiometry throughout. However, the tungsten oxynitride films exhibit modifications in their optical properties (refractive index and optical band gap) with respect to the degree of nitrogen inclusion. The substitution of nitrogen into the metal oxide matrix increases the refractive index, as observed in general for metal oxides ${ }^{57-59}$ including tungsten oxide. ${ }^{60}$ However, the optical bandgap decreases because of the shifts in the absorption edges at long and short wavelengths due to the mixing of nitrogen $2 p$ states with oxygen $2 p$ states. ${ }^{57,61}$

Figure 7 shows the leakage current through films of thickness $60 \mathrm{~nm}$ for hafnium oxide, tungsten oxide, and tungsten oxynitride. In the case of hafnium oxide, the leakage current increases steadily with increasing pulse length for pulse lengths greater than $140 \mu \mathrm{s}$. The leakage current is attributed to the increased carrier density associated with the increasing metallic fraction in the increasingly sub-stoichiometric films. The tungsten oxide films show only weak dependence of leakage current on pulse length across all pulse lengths despite the changes in the refractive index observed. For the tungsten oxynitride films, there is an increasing degradation in the leakage current to higher values with increasing pulse length beyond $160 \mu \mathrm{s}$, correlated with the introduction of defects associated with increasing nitrogen incorporation.

\section{DISCUSSION}

The pulse length dependences observed for target current, optical emission spectra from the plasma, and the film properties suggest that the composition of the target surface is dependent on the HiPIMS pulse length. The differences in behaviour of the Hf and $\mathrm{W}$ targets can be understood in terms of differences in the properties of the metals and their oxides/nitrides. The secondary electron emission and the 


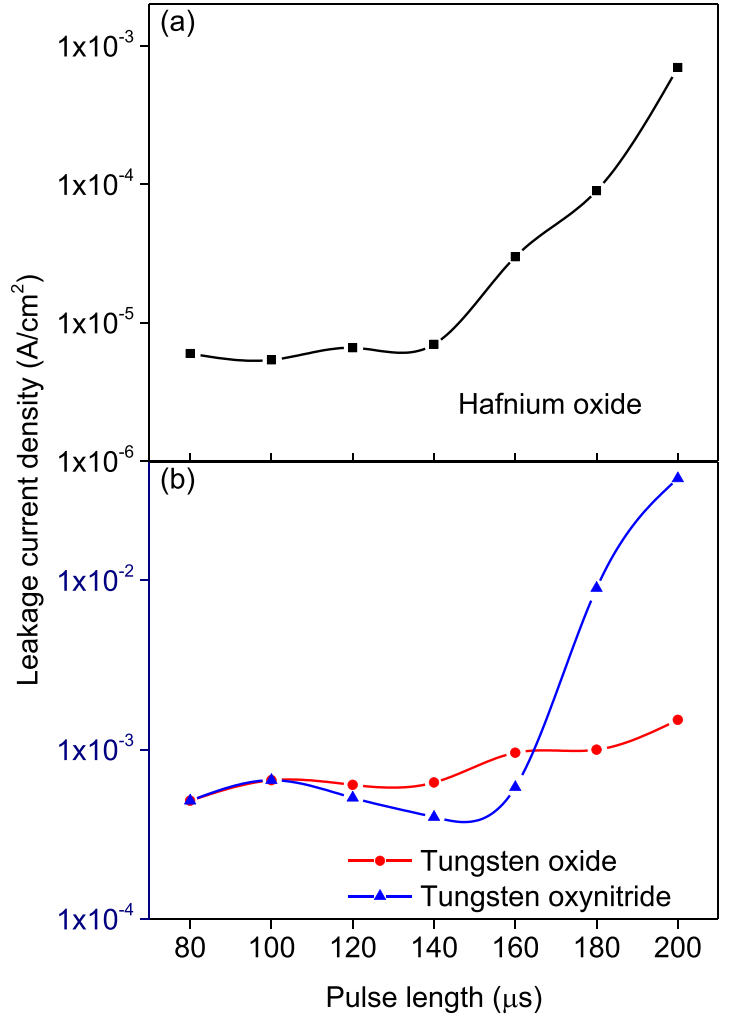

FIG. 7. The leakage current density through the film for an applied probe electric field of $0.1 \mathrm{MV} / \mathrm{cm}$ for (a) hafnium oxide and (b) tungsten oxide and tungsten oxynitride as a function of pulse length.

melting point of pure hafnium metal are lower than those of its oxide, ${ }^{62,63}$ whereas the melting point of tungsten is higher than that of its oxide. ${ }^{63}$ The melting point of tungsten oxide increases with the incorporation of nitrogen, a result expected because the melting point of nitrides is generally higher than that of their corresponding oxides. ${ }^{63}$ Such differences of a target surface layer would be expected to change significantly the properties of the discharge, and we propose a phenomenological model along these lines. Figure 8 shows a schematic diagram of proposed changes to the target surface, depending on the length of the HiPIMS pulse. The images show target conditions at the start and at the end of HiPIMS pulses of various lengths. The composition of the target surface layer is determined by the pulse length and frequency and reaches a cyclic equilibrium wherein the composition is the same at the beginning and end of each pulse, being progressively eroded throughout the pulse and reformed during the after-glow between pulses. In the case of the Hf target (Figure 8(b)), long pulses of $200 \mu \mathrm{s}$ at $150 \mathrm{~Hz}$ etch the hafnium oxide compound layer back to the point where the surface becomes metal rich. However, at pulses shorter than $140 \mu \mathrm{s}$, the oxide layer on the target is retained throughout the pulse. This behavior explains the transition from stoichiometric oxide films at pulses lower than $160 \mu$ s to films progressively richer in metal as the pulse length increases beyond this point. ${ }^{38,40}$ In contrast, the $\mathrm{W}$ target (see Figure 8(c)), which has a higher affinity for oxygen, ${ }^{64,65}$ retains sufficient oxide to maintain identical discharge conditions up to the longest pulses (highest duty cycles) used in this experiment $(200 \mu \mathrm{s}$ at $150 \mathrm{~Hz})$. The

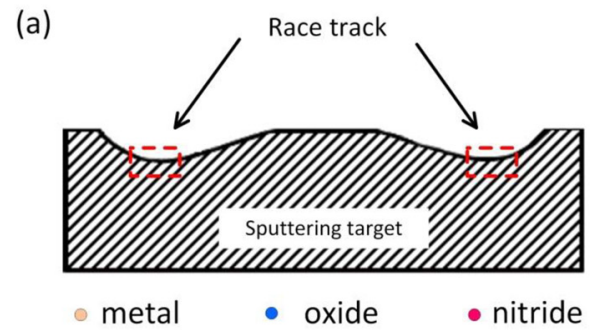

(b)
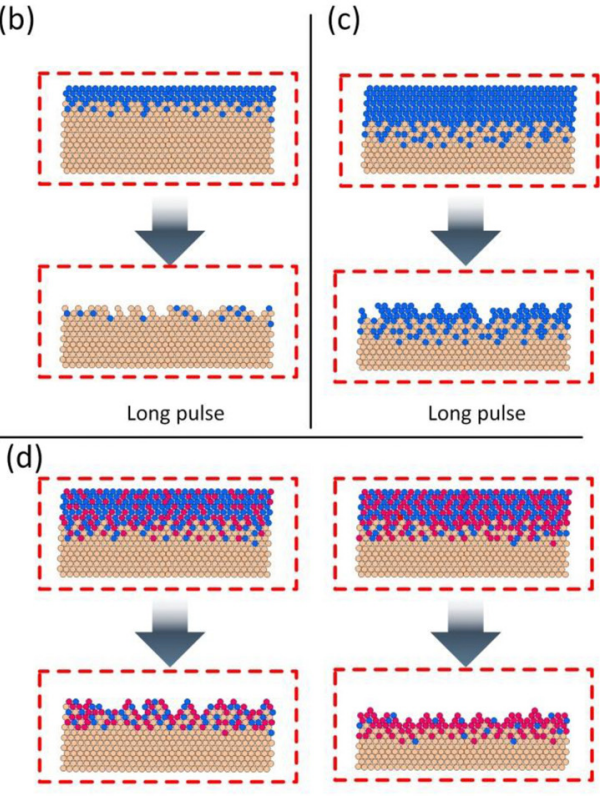

Short pulse
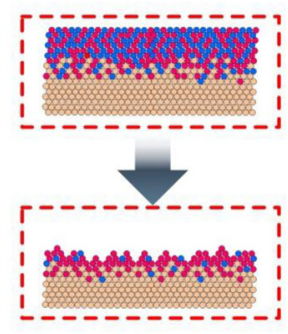

Long pulse

FIG. 8. Schematic diagram showing depth profiles of the race track region (a). The surface condition in the race track is indicated for (b) hafnium in oxygen reactive gas, (c) tungsten in oxygen reactive gas, and (d) tungsten in an oxygen and nitrogen sputtering gas mixture. In each case (b)-(d), the upper image represents the target surface condition at the beginning of the HiPIMS pulse and the lower image represents the condition at the end of the pulse.

melting point of tungsten trioxide $\left(1473^{\circ} \mathrm{C}\right)$ is much lower than that of the tungsten metal $\left(3422^{\circ} \mathrm{C}\right)$, whereas the melting point of hafnium oxide $\left(2758^{\circ} \mathrm{C}\right)$ is higher than that of the hafnium metal $\left(2231^{\circ} \mathrm{C}\right) .^{63}$ The low melting point and low sublimation temperature of $\mathrm{WO}_{3}$ allow the formation of tungsten oxide on the target surface to proceed at a high rate, facilitated by enhanced diffusion of oxygen through the oxide layer. This is consistent with the much higher formation rate of oxide for tungsten $\left(17.57 \mathrm{mg} / \mathrm{cm}^{2}\right.$ at $900^{\circ} \mathrm{C}$ for $120 \mathrm{~min})^{66}$ as compared to hafnium $\left(<5 \mathrm{mg} / \mathrm{cm}^{2}\right.$ at $900^{\circ} \mathrm{C}$ for $120 \mathrm{~min}){ }^{67,68}$ Consequently, the oxide coverage is maintained even when substantial etching occurs during long pulses, so that stoichiometric films are deposited under all conditions tested. In the sputtering process, the thickness of the oxide layer formed on the tungsten target has been reported to be significantly greater than that formed on other metallic targets. ${ }^{69}$

This model is further supported by ellipsometry measurements calculated as an average of 20 points on the target racetrack, fitted using Cauchy layers to determine the compound layer thicknesses. The thickness of the oxide layer on the tungsten target surface is $10.5 \pm 1.3 \mathrm{~nm}$ when measured after being run with $80 \mu$ s pulses, whereas a measurement 


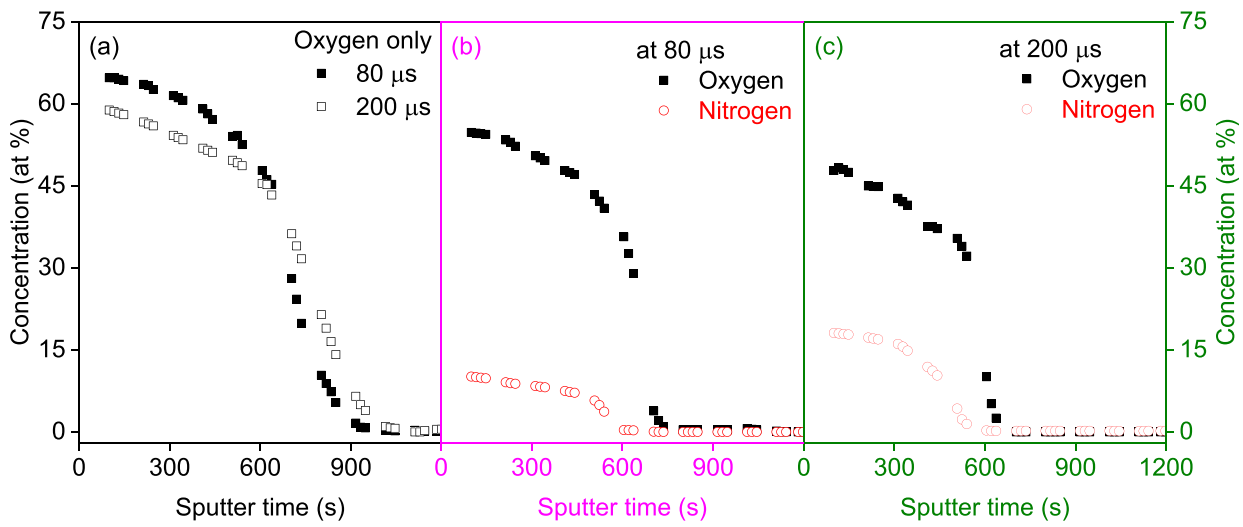

FIG. 9. XPS depth profile taken in the racetrack of a tungsten target after being operated (a) in argon and oxygen and (b) and (c) in an argon, nitrogen, and oxygen gas mixture for short (b) and long (c) pulses. In the case of the argon, reactive oxygen, and nitrogen gas mixture, the flow rate ratio was 10:5:5 sccm, respectively.

after erosion with $200 \mu$ s pulses gives only $6.3 \pm 0.9 \mathrm{~nm}$. In the case of reactive HiPIMS of hafnium, the thickness of oxide layers on the target is $4.9 \pm 0.85 \mathrm{~nm}$ and $1.3 \pm 0.85 \mathrm{~nm}$ for $80 \mu$ s and $200 \mu$ s pulse operation, respectively. An oxide layer of $1.3 \mathrm{~nm}$ at the start of the pulse is so thin that it will be eroded to metal during the pulse, hence producing a metal-rich plasma and metal rich films, as observed.

When nitrogen is added to the process, the properties of the oxynitride compound layer on the target surface lead to strong pulse length dependence. Figure $8(\mathrm{~d})$ shows
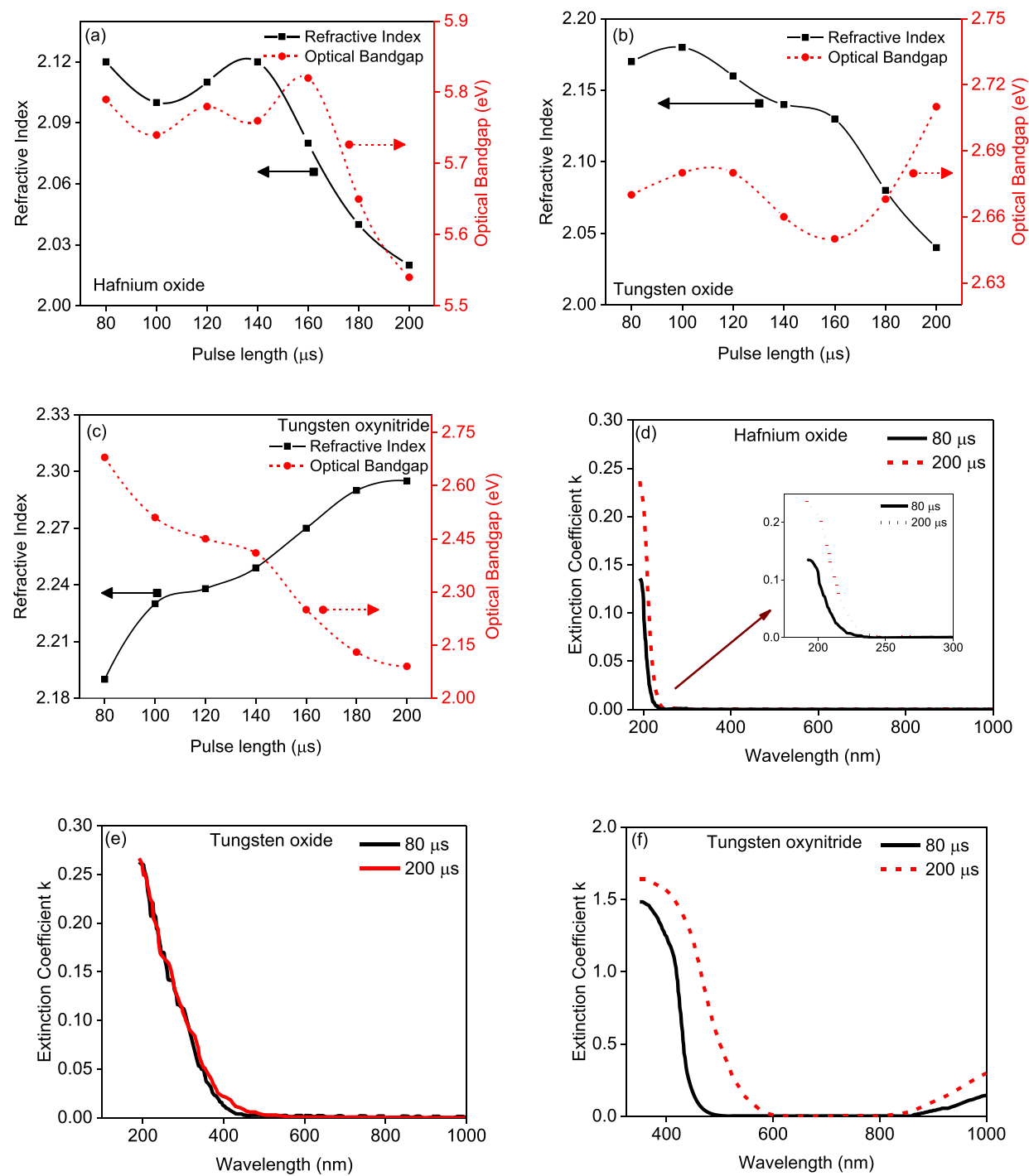

FIG. 10. The refractive index $(500 \mathrm{~nm})$ and the optical bandgap measured for the (a) hafnium oxide, (b) tungsten oxide, and (c) tungsten oxynitride films as a function of pulse length. The extinction coefficient of (d) hafnium oxide, (e) tungsten oxide, and (f) tungsten oxynitride films as a function of wavelength for pulse lengths as indicated in the legend. For tungsten oxide films, the refractive index decreases with increasing pulse length, while there is no variation in extinction coefficient, whereas for tungsten oxynitride, the refractive index increases with an increase in pulse length. 
schematically the effect of short and long pulse operation of a $\mathrm{W}$ target in an oxygen/nitrogen atmosphere. Since the melting point of tungsten nitride $\mathrm{e}^{70,71}$ is higher than that of tungsten oxide $\mathrm{e}^{72,73}$ and the nitride is much less volatile then the oxide,$^{74}$ the nitride component is removed at a slower rate. Previous work has reported an evaporation-assisted sputtering process ${ }^{69}$ for reactive HiPIMS of $\mathrm{WO}_{3}$ that occurs due to its high volatility. Additionally, the surface binding energy for oxygen on a metallic tungsten surface ${ }^{75}$ is lower than that of nitrogen. ${ }^{76}$ Taken together, this means that the target compound layer becomes enriched in nitrogen for long pulse operation relative to that at short pulses. Hence, the plasma discharge and the films deposited at long duty cycles are enriched in nitrogen relative to those deposited at lower duty cycles in the same reactive gas mixture (Figures 3 and 4).

XPS depth profiling (Figure 9) confirms the composition changes as predicted by the model. Although the compositions of the target and deposited films follow the same trends, the target is relatively enriched in nitrogen. Severin et al. ${ }^{77}$ also reported a similar outcome where oxygen is preferentially incorporated in the films, and nitrogen was substantially reduced for relatively small increments in the oxygen flow rate.

The composition of oxygen in the target racetrack operated in $\mathrm{Ar} / \mathrm{O}_{2}$ HiPIMS of tungsten (Figure 9(a)) suggests a slight increase in the oxygen concentration at the surface and a slightly thinner oxide layer for the short pulse length $(80 \mu \mathrm{s})$ compared to the long pulse length $(200 \mu \mathrm{s})$. However, this slight variation could also be explained by slight run-to-run differences in the depth resolution of the sputter etching. Figures 9(b) and 9(c) show the nitrogen and oxygen depth profiles for short $(80 \mu \mathrm{s})$ and long $(200 \mu \mathrm{s})$ pulse lengths. As presented by our phenomenological model, nitrogen is enriched relative to oxygen in the target compound layer for the longer pulses operating in the same gas mixture.

The duty cycle control of reactive HiPIMS processes suggested by the model opens the possibility of creating graded optical properties as a function of film thickness. As shown in Figure 10, varying the duty cycle in HiPIMS allows selection of the real part of the refractive index in both $\mathrm{Hf}$ and $\mathrm{W}$ oxides without significantly increasing the extinction coefficient. This suggests the potential for using in-situ variation of the duty cycle during a single deposition to create graded and/or multilayer optically functional coatings. The pulse length is an easily adjustable parameter that can be varied during a deposition process. In-situ ellipsometry using the Arwin and Aspnes method of fitting presents a highly effective tool to monitor the grading in real time. ${ }^{42}$

\section{CONCLUSION}

We have shown the ability to tune optical and electrical properties of hafnium oxide, tungsten oxide, and tungsten oxynitride coatings through varying the HiPIMS pulse length and therefore the duty cycle. It was proposed that the widely different melting points and volatilities of compound layers formed on target surfaces result in differences in their removal and formation behavior. We have presented a model that explains the pulse length dependent variations observed in target current, optical emission spectra, and film characteristics. The model describes how the target surface layer attains a cyclic equilibrium varying between starting and ending compositions that are determined by a duty cycle dependent balance between erosion and compound formation. While the hafnium oxide compound on the target surface was almost completely etched at relatively long pulses, the tungsten target showed identical discharge conditions for the entire range of pulse lengths studied showing that the oxide layer was still present at the end of the pulse. When a mixture of nitrogen and oxygen was used as the reactive atmosphere, the properties of the oxynitride compound layer on the target surface lead to a marked pulse length dependence. The etch-resistant nitride component became enriched on the target surface during long pulse operation and resulted in the deposition of nitrogen-rich coatings relative to short pulse operation. The changes in surface chemistry of a HiPIMS target, obtained by tuning the pulse characteristics, hold great promise for the fabrication of multilayer films with through thickness graded properties. By varying the pulse length during the deposition process, the beginning and end points of the target surface layer can be modified, leading to controlled modulation of film properties with depth in the deposited film.

\section{ACKNOWLEDGMENTS}

We acknowledge the Australian Research Council for financial support of this work.

\footnotetext{
${ }^{1}$ A. Anders, Surf. Coat. Technol. 204, 2864 (2010).

${ }^{2}$ A. Anders, Surf. Coat. Technol. 257, 308 (2014).

${ }^{3}$ A. von Keudell, A. Hecimovic, and C. Maszl, Contrib. Plasma Phys. 56, 918 (2016).

${ }^{4}$ J. T. Gudmundsson, N. Brenning, D. Lundin, and U. Helmersson, J. Vac. Sci. Technol. A 30, 030801 (2012).

${ }^{5}$ J. Gudmundsson, Vacuum 84, 1360 (2010).

${ }^{6}$ J. T. Gudmundsson, J. Phys.: Conf. Ser. 100, 082013 (2008).

${ }^{7}$ A. E. Ross, R. Sanginés, B. Treverrow, M. M. M. Bilek, and D. R. McKenzie, Plasma Sources Sci. Technol. 20, 035021 (2011).

${ }^{8}$ A. Anders, J. Andersson, and A. Ehiasarian, J. Appl. Phys. 102, 113303 (2007).

${ }^{9}$ J. Andersson and A. Anders, Phys. Rev. Lett. 102, 045003 (2009).

${ }^{10}$ D. Horwat and A. Anders, Appl. Phys. Lett. 97, 221501 (2010).

${ }^{11}$ R. Ganesan, D. McCulloch, N. Marks, M. Tucker, J. Partridge, M. Bilek, and D. McKenzie, J. Phys. D: Appl. Phys. 48, 442001 (2015).

${ }^{12}$ M. Tucker, R. Ganesan, D. McCulloch, J. Partridge, M. Stueber, S. Ulrich, M. Bilek, D. McKenzie, and N. Marks, J. Appl. Phys. 119, 155303 (2016).

${ }^{13}$ M. Mickan, U. Helmersson, H. Rinnert, J. Ghanbaja, D. Muller, and D. Horwat, Sol. Energy Mater. Sol. Cells 157, 742 (2016).

${ }^{14}$ R. Ganesan, B. Murdoch, J. Partridge, S. Bathgate, B. Treverrow, X. Dong, A. Ross, D. McCulloch, D. McKenzie, and M. Bilek, Appl. Surf. Sci. 365, 336 (2016)

${ }^{15}$ B. Murdoch, R. Ganesan, D. McKenzie, M. Bilek, D. McCulloch, and J. Partridge, Appl. Phys. Lett. 107, 112903 (2015).

${ }^{16}$ B. Murdoch, D. McCulloch, R. Ganesan, D. McKenzie, M. Bilek, and J. Partridge, Appl. Phys. Lett. 108, 143504 (2016).

${ }^{17}$ F. Magnus, O. Sveinsson, S. Olafsson, and J. T. Gudmundsson, J. Appl. Phys. 110, 083306 (2011)

${ }^{18}$ J. T. Gudmundsson, Plasma Phys. Controlled Fusion 58, 014002 (2016).

${ }^{19}$ M. Hála, J. Čapek, O. Zabeida, J. Klemberg-Sapieha, and L. Martinu, J. Phys. D: Appl. Phys. 45, 055204 (2012).

${ }^{20}$ D. Horwat, M. Mickan, and W. Chamorro, Phys. Status Solidi C 13, 951 (2016).

${ }^{21}$ S. Zenkin, A. Belosludtsev, Š. Kos, R. Čerstvý, S. Haviar, and M. Netrvalová, Appl. Phys. Lett. 108, 231602 (2016).
} 
${ }^{22}$ J. Vlček, A. Belosludtsev, J. Rezek, J. Houška, J. Čapek, R. Čerstvý, and S. Haviar, Surf. Coat. Technol. 290, 58 (2016).

${ }^{23}$ T. Kozák and J. Vlček, J. Phys. D: Appl. Phys. 49, 055202 (2016).

${ }^{24}$ M. Hala, R. Vernhes, J.-P. Fortier, B. Baloukas, O. Zabeida, J. E Klemberg-Sapieha, L. E. D. T. M. Martinu, and D. Ristau, "Passive and active optical coatings prepared by HiPIMS," in Proceedings of Optical Interference Coatings, Whistler, Canada (Optical Society of America, 2013), p. WC.8.

${ }^{25}$ M. Hála, R. Vernhes, O. Zabeida, J.-E. Klemberg-Sapieha, and L. Martinu, J. Appl. Phys. 116, 213302 (2014).

${ }^{26}$ S. K. Nandi, X. Liu, S. Li, D. K. Venkatachalam, K. Belay, and R. G. Elliman, Behaviour 3, 4 (2014).

${ }^{27}$ S. K. Nandi, X. Liu, S. Li, D. K. Venkatachalam, K. Belay, and R. G. Elliman, "Resistive switching behavior in $\mathrm{HfO}_{2}$ with $\mathrm{Nb}$ as an oxygen exchange layer," Conference on Optoelectronic and Microelectronic Materials \& Devices, Perth, WA (2014), pp. 290-293.

${ }^{28}$ J. Choi, Y. Mao, and J. Chang, Mater. Sci. Eng. R 72, 97 (2011).

${ }^{29}$ A. Palla-Papavlu, M. Filipescu, C. W. Schneider, S. Antohe, P. M. Ossi, G. Radnóczi, M. Dinescu, A. Wokaun, and T. Lippert, J. Phys. D: Appl. Phys. 49, 205101 (2016).

${ }^{30}$ S. Yang and V. K. Agarwal, patent US6631069 B2 (7 October 2003).

${ }^{31}$ A. Subrahmanyam and A. Karuppasamy, Sol. Energy Mater. Sol. Cells 91, 266 (2007).

${ }^{32}$ O. Kartachova, A. M. Glushenkov, Y. Chen, H. Zhang, X. J. Dai, and Y. Chen, J. Power Sources 220, 298 (2012).

${ }^{33}$ D. Depla and R. De Gryse, Surf. Coat. Technol. 183, 184 (2004).

${ }^{34}$ D. Depla and R. De Gryse, Surf. Coat. Technol. 183, 190 (2004).

${ }^{35}$ D. Depla and R. De Gryse, Surf. Coat. Technol. 183, 196 (2004).

${ }^{36}$ S. Berg, H. O. Blom, T. Larsson, and C. Nender, J. Vac. Sci. Technol. A 5, 202 (1987).

${ }^{37}$ T. Kubart, T. Polcar, O. Kappertz, N. Parreira, T. Nyberg, S. Berg, and A. Cavaleiro, Plasma Process. Polym. 4, S522 (2007).

${ }^{38}$ R. Ganesan, B. J. Murdoch, B. Treverrow, A. E. Ross, I. S. Falconer, A. Kondyurin, D. G. McCulloch, J. G. Partridge, D. R. McKenzie, and M. M. M. Bilek, Plasma Sources Sci. Technol. 24, 035015 (2015).

${ }^{39}$ R. Ganesan, B. Treverrow, B. Murdoch, D. Xie, A. E. Ross, J. G. Partridge, I. S. Falconer, D. G. McCulloch, D. R. McKenzie, and M. M. M. Bilek, J. Phys. D: Appl. Phys. 49, 245201 (2016).

${ }^{40}$ A. E. Ross, R. Ganesan, M. M. M. Bilek, and D. R. McKenzie, Plasma Sources Sci. Technol. 24, 025018 (2015).

${ }^{41}$ H. Arwin and D. E. Aspnes, Thin Solid Films 113, 101 (1984).

${ }^{42}$ L. Ryves, M. M. M. Bilek, T. W. H. Oates, R. N. Tarrant, D. R. McKenzie, F. A. Burgmann, and D. G. McCulloch, Thin Solid Films 482, 133 (2005).

${ }^{43}$ T. W. H. Oates, D. R. McKenzie, and M. M. M. Bilek, Phys. Rev. B 70, 195406 (2004).

${ }^{44}$ J. Tauc, R. Grigorovici, and A. Vancu, Phys. Status Solidi B 15, 627 (1966).

${ }^{45}$ G. C. A. M. Janssen, M. M. Abdalla, F. van Keulen, B. R. Pujada, and B. van Venrooy, Thin Solid Films 517, 1858 (2009).

${ }^{46}$ R. M. Chaudhria and A. W. Khan, Proc. Natl. Acad. Sci. 7, 197 (1941), available at http://www.insa.nic.in/writereaddata/UpLoadedFiles/PINSA/ Vol07_1941_2_Art08.pdf.

${ }^{47}$ A. Huang, P. K. Chu, and Z. Yang, Hafnium-Based High-k Gate Dielectrics (INTECH Open Access Publisher, 2010).
${ }^{48}$ B. Chowdari, H. Yoo, G. Choi, and J. Lee, Solid State Ionics: The Science and Technology of Ions in Motion (World Scientific, 2004).

${ }^{49}$ Y. G. Shen, Y. W. Mai, D. R. McKenzie, Q. C. Zhang, W. D. McFall, and W. E. McBride, J. Appl. Phys. 88, 1380 (2000).

${ }^{50}$ B. Abendroth, H. U. Jäger, W. Möller, and M. Bilek, Appl. Phys. Lett. 90, 181910 (2007).

${ }^{51}$ M. Bowes, P. Poolcharuansin, and J. W. Bradley, J. Phys. D: Appl. Phys. 46, 045204 (2013).

${ }^{52}$ C. Maszl, W. Breilmann, J. Benedikt, and A. v. Keudell, J. Phys. D: Appl. Phys. 47, 224002 (2014).

${ }^{53}$ M. Wen, Q. N. Meng, W. X. Yu, W. T. Zheng, S. X. Mao, and M. J. Hua, Surf. Coat. Technol. 205, 1953 (2010).

${ }^{54}$ T. Polcar and A. Cavaleiro, Int. J. Refract. Met. Hard Mater. 28, 15 (2010).

${ }^{55}$ L. Maillé, C. Sant, P. Aubert, and P. Garnier, Thin Solid Films 479, 201 (2005).

${ }^{56}$ N. M. G. Parreira, N. J. M. Carvalho, and A. Cavaleiro, Thin Solid Films 510, 191 (2006)

${ }^{57}$ S. H. Mohamed, O. Kappertz, J. M. Ngaruiya, T. Niemeier, R. Drese, R. Detemple, M. M. Wakkad, and M. Wuttig, Phys. Status Solidi A 201, 90 (2004).

${ }^{58}$ J. M. Ngaruiya, O. Kappertz, C. Liesch, P. Müller, R. Dronskowski, and M. Wuttig, Phys. Status Solidi A 201, 967 (2004)

${ }^{59}$ S. Venkataraj, D. Severin, S. H. Mohamed, J. Ngaruiya, O. Kappertz, and M. Wuttig, Thin Solid Films 502, 228 (2006).

${ }^{60}$ O. R. Nunez, A. J. Moreno Tarango, N. R. Murphy, L. C. Phinney, K. Hossain, and C. V. Ramana, Thin Solid Films 596, 160 (2015).

${ }^{61}$ M. Ohring, Materials Science of Thin Films (Academic Press, 2001).

${ }^{62} \mathrm{G}$. V. Samsonov, The Oxide Handbook (Springer Science \& Business Media, 2013).

${ }^{63}$ D. L. Perry, Handbook of Inorganic Compounds (CRC Press, 2016).

${ }^{64}$ J. A. Becker, E. J. Becker, and R. G. Brandes, J. Appl. Phys. 32, 411 (1961).

${ }^{65}$ E. A. Gulbransen, K. F. Andrew, and F. A. Brassart, J. Electrochem. Soc. 111, 103 (1964).

${ }^{66} \mathrm{~J}$. Wendel, Diploma thesis, Lund University, Lund, 2014.

${ }^{67}$ W. W. Smeltzer and M. T. Simnad, Acta Metall. 5, 328 (1957).

${ }^{68} \mathrm{P}$. Kofstad and S. Espevik, J. Less Common Met. 12, 382 (1967).

${ }^{69}$ A. Hemberg, J.-P. Dauchot, R. Snyders, and S. Konstantinidis, J. Vac. Sci. Technol. A 30, 040604 (2012).

${ }^{70} \mathrm{~B}$. Predel, in $L i-M g-N d-Z r$, edited by O. Madelung (Springer, Berlin, Heidelberg, 1997), p. 1.

${ }^{71}$ H. A. Wriedt, Bull. Alloy Phase Diagrams 10, 358 (1989).

${ }^{72} \mathrm{~B}$. Predel, in $\mathrm{Ni}-\mathrm{Np}-\mathrm{Pt}-\mathrm{Zr}$, edited by O. Madelung (Springer, Berlin, Heidelberg, 1998), p. 1.

${ }^{73}$ H. A. Wriedt, Bull. Alloy Phase Diagrams 10, 368 (1989).

${ }^{74}$ N. A. Toropov and V. P. Barzakovskii, in High-Temperature Chemistry of Silicates and Other Oxide Systems/Vysokotemperaturnaya Khimiya Silikatnykh I Drugikh Okisnykh Sistem/Вьісокотетпераtyphaя Хития Силикаthьіх И Других Окисhьіх | Сисtет (Springer, Boston, MA, USA, 1966), p. 177.

${ }^{75}$ C. M. Varma and A. J. Wilson, Phys. Rev. B 22, 3795 (1980).

${ }^{76}$ K. Schmid, A. Manhard, C. Linsmeier, A. Wiltner, T. Schwarz-Selinger, W. Jacob, and S. Mändl, Nucl. Fusion 50, 025006 (2010).

${ }^{77}$ D. Severin, K. Sarakinos, O. Kappertz, A. Pflug, and M. Wuttig, J. Appl. Phys. 103, 083306 (2008) 